\title{
Drosophila melanogaster chemical ecology revisited: 2-D distribution maps of sex pheromones on whole virgin and mated flies by mass spectrometry imaging
}

Filip Kaftan ${ }^{1 *}$, Purva Kulkarni ${ }^{2}$, Markus Knaden ${ }^{1}$, Sebastian Böcker², Bill S. Hansson ${ }^{1}$ and Aleš Svatoš ${ }^{* *}$

\begin{abstract}
Background: Sexual behavior in Drosophila melanogaster flies is greatly influenced by chemical cues. In this study, a spatial distribution of female and male sex pheromones was investigated on the surface of virgin and mated sixday-old fruit flies. Surface analysis was performed using the technique of mass spectrometry imaging (MSI) matrixassisted laser desorption/ionization - time of flight (MALDI-TOF) and confirmed by gas chromatography - mass spectrometry (GC-MS) analysis of hexane extracts prepared from dissected flies.
\end{abstract}

Results: MALDI-TOF MSI experiments focused on female pheromones (7Z,11Z)-heptacosa-7,11-diene (7,11-HD) and (7Z,11Z)-nonacosa-7,11-diene (7,11-ND) were enhanced by using lithium 2,5-dihydroxybenzoate (LiDHB) matrix to improve the ionization and quality of MS spectra. Oxygenated compounds represented by male anti-attractants 11cis-vaccenyl acetate (cVA) and 3-O-acetyl-1,3-dihydroxy-octacosa-11,19-diene (CH503) were successfully ionized without MALDI matrix in the form of potassium adducts in laser desorption ionization (LDI-TOF MSI) mode. A similar pattern of distribution for 7,11-HD and 7,11-ND was observed on the surface of both the left and the right female wing, with the strongest signals at the base of the wing. 7,11-HD and 7,11-ND were additionally detected on female legs but not on the body. The distribution of both male pheromones, CVA and CH503, was localized in virgin male flies solely on the abdominal tip and anogenital region. In addition, results from MSI experiments with mated flies showed the distribution of CVA and $\mathrm{CH} 503$ also on the female abdomen and thorax, demonstrating that anti-attractants were transferred from males to females during copulation. Results from LDI/MALDI-TOF MSI were supported by GC-MS analysis of hexane extracts prepared from different parts of virgin male or female Drosophila flies. Similar amounts of 7,11-HD and 7,11-ND were present on the legs, body and wings (127 $\pm 5 \mathrm{ng}$ and $170 \pm 8 \mathrm{ng}$, respectively). cVA was detected only on the male body. All acquired MSI datasets were affected by mass shift (predominantly between $\pm 0.2 \mathrm{Da}$ to $\pm 0.4 \mathrm{Da}$ ), which was reduced using a mass recalibration approach.

* Correspondence: filip.kaftan@gmail.com; svatos@ice.mpg.de

${ }^{1}$ Max Planck Institute for Chemical Ecology, Hans-Knöll-Strasse 8, D-07745 Jena, Germany

Full list of author information is available at the end of the article

(c) The Author(s). 2020 Open Access This article is licensed under a Creative Commons Attribution 4.0 International License, which permits use, sharing, adaptation, distribution and reproduction in any medium or format, as long as you give appropriate credit to the original author(s) and the source, provide a link to the Creative Commons licence, and indicate if changes were made. The images or other third party material in this article are included in the article's Creative Commons licence, unless indicated otherwise in a credit line to the material. If material is not included in the article's Creative Commons licence and your intended use is not permitted by statutory regulation or exceeds the permitted use, you will need to obtain permission directly from the copyright holder. To view a copy of this licence, visit http://creativecommons.org/licenses/by/4.0/ The Creative Commons Public Domain Dedication waiver (http://creativecommons.org/publicdomain/zero/1.0/) applies to the data made available in this article, unless otherwise stated in a credit line to the data. 
(Continued from previous page)

Conclusions: The LDI/MALDI-TOF MSI technique makes it possible to study the distribution of female and male sexual pheromones on D. melanogaster flies. Moreover, the technique enables the transfer of male sex pheromones to females during copulation to be visualized. However, imaging experiments of 3-D biological samples performed on a single TOF-MS instrument equipped with a MALDI ion source and UV nitrogen laser evinced a photo-electric charging/discharging, a phenomenon that often leads to unpredictable mass shifts and poor mass accuracy.

Keywords: MALDI-TOF, Laser desorption, Mass spectrometry imaging, Hexane extracts, GC-MS, Behavior, Drosophila melanogaster, Sex pheromones, CVA, CH503, 7,11-HD, 7,11-ND

\section{Background}

Sexual behavior in Drosophila melanogaster flies is influenced by non-chemical and chemical cues. Non-chemical signals include visual and acoustic stimuli; for example, male flies visually track the movement of females $[1,2]$ and produce a courtship song by vibrating one or both wings to initiate mating [3-5]. However sounds can also be emitted in the same way by females in order to attract males [6]. Although the auditory component of the wing-vibration song has been thoroughly investigated [7], whether wing vibration is also involved in the dispersal of pheromones is unknown.

Chemical cues in Drosophila include sex pheromone signals. Volatile pheromones in Drosophila [8-10] as well as close-range sex pheromones in D. melanogaster are well known, including hydrocarbons and oxygenated hydrocarbons that can be perceived either by olfactory organs, such as antennae or maxillary palps; by contact with the tarsae; or by gustatory receptors on the proboscis [11, 12]. One of the best-known Drosophila sex pheromones is 11-cis-vaccenyl acetate (cVA), which plays a dual role as a short-lived inhibitor of male courtship and as an aggregation pheromone [13-15]. Furthermore, cVA is a male pheromone that is synthesized in the ejaculatory bulb [13] and transferred from males to females during mating [16]. The presence of cVA on a mated female has been shown to reduce its attractiveness to males for several hours [17]. Another D. melanogaster male sex pheromone, 3-Oacetyl-1,3-dihydroxy-octacosa-11,19-diene ( $\mathrm{CH} 503), \quad$ is also an inhibitor of male courtship. Unlike cVA, CH503 remains on the female cuticle for more than 10 days [18]. In addition to male-specific pheromones, the femalespecific sex pheromones (7Z,11Z)-heptacosa-7,11-diene (7,11-HD) and (7Z,11Z)-nonacosa-7,11-diene (7,11-ND) are both thought to serve as attractants for males [19].

Mass spectrometry imaging (MSI) is a method for visualizing the two-dimensional (2-D) spatial distribution of compounds of interest across a sample's entire imaged area. MSI provides a rastering of the sample line by line and simultaneously desorbs/ionizes its surface, typically using an ultraviolet laser and MALDI technology. In order to enhance the quality of MS spectra and to ionize specific groups of compounds, a MALDI matrix is applied on the sample [20].
Compared with conventional techniques used to detect pheromones or semiochemicals, MSI provides not only a 2-D distribution of the desired compound over the entire surface of the analyzed sample but also (via MS signal intensity) its relative quantity within a lateral distribution. This information can be obtained for a hundreds of different compounds in a single MSI experiment. When MSI is applied to a Drosophila fly sample, a 2-D image can be constructed for particular $m / z$ ratios; these ratios can represent chemical cues present on a fly cuticle [20].

In our previous studies, we have shown a presence of 7,11-HD and 7,11-ND on Drosophila melanogaster female wings [21] and the presence of cVA on males [22]. These data were acquired and used in order to demonstrate the utility of the LiDHB matrix [23] and the usefulness of a newly developed MSI data recalibration method [24]. We now hope to use these results to help address long-standing biological questions [8, 25].

Although the behavioral and physiological functions of the different pheromones are well understood, in this study, we describe their detailed 2-D distribution on the body surface of $D$. melanogaster and link the spatial occurrence of the pheromones with the fly's sexual behavior. Here we discuss the pros and cons of applying the MALDI-TOF MSI method to study questions in chemical ecology.

To support our MSI data, we dissected the legs, wing and body parts of entire flies, after briefly washing them in hexane, and quantified the surface lipids via GC-MS analysis.

\section{Methods}

\section{Insect material}

D. melanogaster (Meigen, 1830) flies (Diptera, Drosophilidae) were obtained from a colony (strain Canton S) maintained at the Max Planck Institute for Chemical Ecology in Jena, Germany. We analyzed 6-day-old flies, each of which was bred separately (isolated at the pupal stage $)$ in an Eppendorf tube $(2 \mathrm{~mL})$ at $25^{\circ} \mathrm{C}, 70 \%$ humidity and a 12/12 h light darkness circle. Flies were fed for 4 days on a normal diet $(30 \mathrm{mg}$ of solid food - recipe shown in Table S1 - plus a moistened tissue on the bottom of the vial to prevent food from drying out) and for 
the last 2 days on sugar solution only to eliminate food contamination of the fly surface. To obtain mated pairs of fruit flies, a female fly and a male fly were placed in a refrigerator $\left(4^{\circ} \mathrm{C}\right)$ and cooled for approximately $3 \mathrm{~min}$ before being put together into a new Eppendorf tube. The copulation was carried out at laboratory temperature, and pairs were immobilized at $-20^{\circ} \mathrm{C} \mathrm{im-}$ mediately afterward. Virgin flies were immobilized the same way.

Fly samples were warmed slowly (sealed in plastic Eppendorf tubes) to room temperature prior to being fixed onto dedicated MALDI targets.

\section{Chemicals}

2,5-Dihydroxybenzoic acid (DHB) for MALDI MS with purity above $98 \%$ was purchased from Sigma-Aldrich. Lithium 2,5-Dihydroxybenzoate (LiDHB) matrix was synthesized as described previously [23]. High purity MS grade solvents (acetone, ethanol, methanol, dichloromethane) were also supplied by Sigma-Aldrich (Germany) as well as analytic-grade poly (ethylene glycol) oligomers (PEG with average masses of $200 \mathrm{Da}, 300$ $\mathrm{Da}, 600 \mathrm{Da}$ and $1000 \mathrm{Da}$ ) for calibration of the mass spectrometer. 11-cis-vaccenyl acetate (cVA), (7Z,11Z)heptacosa-7,11-diene (7,11-HD) and (7Z,11Z)-nonacosa7,11-diene (7,11-ND) standards were purchased from Cayman Chemicals (Germany).

\section{MALDI imaging}

LDI/MALDI-TOF imaging experiments of D. melanogaster flies were performed on MALDI Micro MX (Waters, UK) pumped down from $1 \times 10^{-6}$ up to $9 \times 10^{-7} \mathrm{mbar}$ and operated in reflectron mode with the acceleration and plate voltages at $-12 \mathrm{kV}$ and $5 \mathrm{kV}$, respectively. Extraction pulse duration was set to 500 ns. Desorption and ionization were achieved using MNL 103-LD pulsed nitrogen laser ( $337.1 \mathrm{~nm}$; pulse half width $3 \mathrm{~ns}$; pulse energy $\geq 90 \mu \mathrm{J}$; repetition rate up to $30 \mathrm{~Hz}$; LTB Lasertechnik Berlin, Germany). Matrix ions were suppressed, and the low mass cut-off was set at $150 \mathrm{Da}$.

Intact flies were fixed into complex profiled cells imprinted on a dedicated aluminum MALDI plate [20] using two-part resin epoxy glue (Hardman ${ }^{\circ}$, www.royaladhesives.com, Belleville, NJ, USA). LiDHB matrix solution was prepared in acetone:dichloromethane $(9: 1, \mathrm{v} / \mathrm{v})$ at a concentration of $20 \mathrm{mg} / \mathrm{mL}$. The matrix was sprayed on fly samples before drying by a commercial airbrush (Harder \& Steenbeck, www.airbrushuniverse.com, Norderstedt, Germany) with a $0.15 \mathrm{~mm}$ diameter nozzle from a distance of $160 \mathrm{~mm}$. For one sample, $2 \mathrm{~mL}$ of LiDHB matrix solution was used to form approximately 60 layers. LiDHB matrix prepared in $\mathrm{MeOH}(20 \mathrm{mg} / \mathrm{mL})$ was applied on fixed female wings and legs using SunChrom MALDI spotter (SunChrom GmbH, Germany) and this spraying method: line distance $2 \mathrm{~mm}$, spraying speed 800 $\mathrm{mm} / \mathrm{min}$ with $5 \mathrm{~s}$ drying time and with a flow rate of $60 \mu \mathrm{L} / \mathrm{min}$. After matrix application, whole intact fly samples were placed into a desiccator with solid potassium hydroxide at ambient temperature and pressure for $6 \mathrm{~h}$; dissected wings and legs, $1 \mathrm{~h}$. After that time, the samples were completely dry and ready for imaging experiments.

Samples of flies were imaged using a step size of $100 \mu \mathrm{m}$. The number of laser shots per spot was optimized and set to $120(60 \mu \mathrm{J} / \mathrm{shot})$ at a laser repetition rate of $15 \mathrm{~Hz}$ for MALDI-TOF MSI experiments $(30 \mathrm{~Hz}$ for the samples of fly legs \& wings) and 40 shots $(60 \mu \mathrm{J} /$ shot) per spot at a laser repetition rate of $5 \mathrm{~Hz}$ for LDITOF MSI. The range of measured masses was set from $100 \mathrm{Da}$ to $1000 \mathrm{Da}$ and $300 \mathrm{Da}$ to $550 \mathrm{Da}$ for the samples of fly legs and wings, respectively. The typical laser ablation spot of $30 \mathrm{~Hz} / 120$ shots $(60 \mu \mathrm{J} / \mathrm{shot})$ is represented by an oval area $40 \mu \mathrm{m} \times 60 \mu \mathrm{m}$ from where most of the energy is focused. The outer ring is $70 \mu \mathrm{m} \times 90 \mu \mathrm{m}$.

Data were acquired with MassLynx 4.0 software and processed with custom-made software MALDI Image Convertor (Waters, UK) to obtain laterally differentiated data. These datasets were then analyzed with a method developed in-house and recalibrated in order to eliminate mass shift. Recalibrated MSI datasets were subsequently converted to 2-D ion intensity maps.

\section{Recalibration of MSI dataset}

The acquired imaging datasets affected by mass shift were imported into MATLAB (MATLAB and Statistics Toolbox Release R2012b, The MathWorks ${ }^{\circ}$, Inc., Natick, Massachusetts, USA) using available protocols. In order to improve the quality of the MS spectra, each dataset was subjected to the following preprocessing steps: baseline correction using the msbackadj MATLAB routine (Bioinformatics Toolbox) and smoothing using mslowess MATLAB routine (Bioinformatics Toolbox). Next, peak-picking was performed using the mspeaks routine available in MATLAB. For every dataset, the peaklists were exported as individual text files along with their coordinate position information.

Peaklists belonging to every dataset were recalibrated using the lock mass-free mass spectra recalibration approach proposed by Kulkarni et al. [24]. To rearrange the order of peaklists for recalibration, out of the two peaklist ordering approaches proposed by Kulkarni et al., the crystal growth (CG) ordering approach was applied. MSICorrect software implementing this recalibration approach can be downloaded from https://bio.informatik. uni-jena.de/software/msicorrect/.

\section{GC-MS analysis of sex pheromones in hexane extracts of D. melanogaster}

The samples were measured on an Agilent 7890A gas chromatograph (Agilent Technologies, CA) running in 
splitless mode and connected to an Agilent 5975C mass spectrometer (electron ionization mode, $70 \mathrm{eV}$, ion source: $230{ }^{\circ} \mathrm{C}$, quadrupole: $150{ }^{\circ} \mathrm{C}$, mass scan range: $\mathrm{m} / \mathrm{z}$ 50-450). For GC-MS analysis, $1 \mu \mathrm{L}$ of each sample was injected on a polar column (HP-INNOWAX, $30 \mathrm{~m}$ length, $0.25 \mathrm{~mm}$ ID, $0.25 \mu \mathrm{m}$ film thickness, J\&W Scientific) which was operated under constant helium flow (1 $\mathrm{mL} / \mathrm{min}$ ). The $\mathrm{GC}$ oven was kept at $40^{\circ} \mathrm{C}$ for $1 \mathrm{~min}$, ramped at $20^{\circ} \mathrm{C} / \mathrm{min}$ to $260{ }^{\circ} \mathrm{C}$ and then kept at $260{ }^{\circ} \mathrm{C}$ for $20 \mathrm{~min}$.

Compounds were identified by comparing mass spectra to authentic reference compounds in our databases, based on predicted fragmentation patterns, or to those published by the National Institute of Standards and Technologies.

\section{Sample preparation of wing hexane extracts of virgin $D$. melanogaster females}

The hexane extracts for GC-MS analysis of male and female pheromones were prepared separately from 5 flies of each sex. For these and all other experiments, we used flies from the Canton $\mathrm{S}$ strain. Male and female flies were cut into three parts: wings, legs, and head/thorax/ abdomen. Micro-scissors and tweezers were carefully cleaned in hexane before every cutting step, and the preparation was performed in such a way as to eliminate any cross-contamination of fly body parts. Fifteen samples obtained from 5 males and 5 females were collected in glass inserts and mixed with $60 \mu \mathrm{L}$ of hexane. After $30 \mathrm{~s}$ of extraction, performed at laboratory temperature, $4 \mu \mathrm{L}$ of internal standard $n$-C16 diluted in hexane at a concentration of a $50 \mathrm{ng} / \mu \mathrm{L}$ was added to the mixture. The supernatant was removed by glass capillary, transferred to a new glass insert and subsequently dried using a gentle stream of argon to a marked level of $20 \mu \mathrm{L}$. The final concentration of $\mathrm{C} 16$ standard for each sample was $10 \mathrm{ng} / \mu \mathrm{L}$.

\section{Sample preparation of anogenital extracts of virgin $D$. melanogaster males}

In contrast to the sample preparation of wing extracts, the anogenital extracts of male fruit flies had to be prepared manually by rubbing specific regions of the male fly body with small pieces of paper. For each experiment, 14 male fruit flies were selected. The anogenital region of each fly was rubbed with blotting paper that had been pre-extracted with hexane and cut with scissors into small pieces $(1 \mathrm{~mm} \times 2 \mathrm{~mm})$. Each piece of paper was soaked in hexane using tweezers and then used to gently wipe the abdominal tip and the genital area. The procedure was repeated twice with new paper for each individual fly. Obtained papers containing extracts of surface lipids were collected in glass inserts and extracted with hexane $(40 \mu \mathrm{L})$. Individual samples were prepared as specified in the description of hexane extracts of wings.

\section{Surface area of virgin D. melanogaster females}

The surface of the whole female fly was measured using a Keyence digital microscope VHX-5000 equipped with a zoom lens VHX-J20T (Keyence Germany GmbH, Germany). Measurements were performed on several six-day-old virgin female flies with a length of $2.5 \mathrm{~mm}$, representing the average size of female flies. First wings, then legs, were cut from the thorax and their dimensions measured. After that, the body of the fly was cut into head, thorax and abdomen, and measured. Obtained dimensions of each part were averaged from three individual female flies, and the surface was calculated using the formula for a cylinder or a cuboid surface area. Based on our measurements, the total surface area of one Drosophila female fly corresponded to $22.55 \mathrm{~mm}^{2}$. The average surface area of female legs was approximately 2.9 $\mathrm{mm}^{2}$, of wings, approximately $11.5 \mathrm{~mm}^{2}$, and of the rest of the fly body surface area, ca $8.2 \mathrm{~mm}^{2}$. For details, see Table S2.

\section{Scanning electron microscopy}

Images of $D$. melanogaster were obtained using a scanning electron microscope (SEM) Zeiss (LEO) 1450VP (Carl Zeiss AG, Germany). The scanning microscope was also used to study the quality of the LiDHB layer deposited on the flies by spraying. The MALDI targets with flies on them were coated with a thin layer $(20 \mathrm{~nm}$ thick) of gold using SCD 050 (Bal-Tec, Liechtenstein) sputter coater. The targets were fixed by conductive carbon double-sided tape (Ted Pella Inc., USA) onto a 3-D holder in the SEM chamber. Images were made using the acceleration voltage of $10 \mathrm{kV}$.

\section{Results}

Dedicated MALDI targets designed for previously published LDI/MALDI-TOF MSI experiments of the whole D. melanogaster flies [20] meet the requirements for experiments presented in this study, including MSI performed on flies which were imaged from ventral side.

The sex pheromones analyzed via LDI-TOF MSI mode were predominantly detected in the form of adducts with potassium and sodium $\left([\mathrm{M}+\mathrm{K}]^{+} ;[\mathrm{M}+\mathrm{Na}]^{+}\right)$cations, both of which are naturally present on the fly cuticle [21, 22, 24].

The mass spectra recalibration process applied to MSI datasets significantly reduced the mass shift [24]. In addition, the quality of MSI allowed us to generate 2-D ion intensity maps; these were improved by manual peak-picking, which was part of the recalibration method that helped to recognize and include even lowintensity MS signals. 


\section{Effect of fly handling, matrix deposition, and drying}

Several experiments using an optical digital microscope and a scanning electron microscope were performed to investigate the potentially negative effects of drying on the chemical composition of the fly cuticle. We wanted to investigate what happens when the fly abdomen lacking the mechanical support provided by our dedicated MALDI target [22] - dries out.

Based on detailed images obtained by SEM, we showed that the abdominal cuticle in particular evinces very high flexibility and compactness under the stress caused by shrinkage related to drying. We did not observe any visible damage, such as cracks or clefts, in the tergites during this process (Fig. S1).

When the quality of the LiDHB layer deposited on the fly via spraying was investigated using SEM, the images showed uniform coverage: the matrix was spread across the entire surface of the wings. Matrix deposition formed by multiple layer depositions using both a manual airbrush, and SunChrom MALDI spotter provided a uniform layer of matrix aggregated in densely packed lens-like isles with ca $30 \mu \mathrm{m}$ diameter and a distance between of ca $50 \mu \mathrm{m}$ (Fig. S2 a, b, c, d). As the laser ablation area is $40 \mu \mathrm{m} \times 60 \mu \mathrm{m}$ and the step size $100 \mu \mathrm{m}$, we consider the matrix deposition to be uniform.

\section{MALDI-TOF MSI of female pheromones on virgin $D$. melanogaster females}

MSI experiments of virgin $D$. melanogaster female flies was expected to reveal the distribution of the sexual pheromones 7,11-HD and 7,11-ND. Unfortunately, ionization and visualization of these hydrocarbons was problematic. The main problem was the volatility of these dienes under vacuum pressure (up to $9.10^{-7}$ mbar). Notably, previously published LDI profiling data acquired under much higher pressure (2-4 mbar) [18] clearly showed adduct ions of these dienes, supporting the usefulness of pressure for detecting the pheromones under LDI conditions. Because it was not possible to work in the LDI-TOF mode, an application of LiDHB MALDI matrix on the flies was necessary to prevent pheromone loss in the vacuum by its co-crystallization with the matrix. Matrix deposition onto the fly surface also helped to increase the ionization of pheromones (Fig. S3). In order to minimize the loss of 7,11-HD and 7,11-ND from the fly cuticle, the matrix was applied on dissected female wings and legs, and intact female flies by spraying immediately after samples were fixed on the MALDI target. The samples were subsequently placed into a desiccator and allowed to dry under atmospheric pressure for $6 \mathrm{~h}$ in the case of whole flies and $1 \mathrm{~h}$ for wings or legs.

Based on the results from MALDI-TOF MSI experiments, the distribution of both female pheromones, 7 , 11-HD and 7,11-ND, was localized on the wings and legs of females; the strongest signals were observed at the base of the wings and on the hindmost two segments of the legs. These signals were symmetrical on both wings of single female flies and were similar among females (Fig. 1). The same distribution of 7,11-HD and 7,11-ND was obtained from the dorsal and ventral side of the wings (data not shown). The ion intensity maps (Fig. 1a, d) corresponding to the preferably formed lithium adduct of 7,11-HD at $m / z$ 383.4 $\left([\mathrm{M}+\mathrm{Li}]^{+}\right)$and 7,11-ND at $m / z$ 411.4 $\left([\mathrm{M}+\mathrm{Li}]^{+}\right)$suggest that both dienes were concentrated at the base of the wings.

In MALDI-TOF MSI of female wings, we chose a mass window of $\pm 0.4 \mathrm{Da}$ to visualize the ion maps, despite the rather planar wings, because the region of the wing base was always elevated (by approximately $300 \mu \mathrm{m}$ ) compared to the rest of the sample. Due to the higher thickness of this wing area, it was the location of a mass shift.

Results from MALDI-TOF MSI of female wings were supported by a GC-MS analysis of hexane extracts of female wings; MS spectra obtained from these analyses confirmed presence of both female pheromones (Fig. S4). Yet the signals from 7,11-HD and 7,11-ND visualized in MSI experiments performed on whole intact female flies were weak (Fig. S3). Despite the fact that we almost did not see hydrocarbons in MSI of whole female flies, there were many signals corresponding to unknown signals (Fig. S5) and symmetrically distributed on the wing base.

\section{Distribution of 7,11-HD and 7,11-ND on legs of virgin females}

Although distribution of female dienes at the base of the wings was unexpected, their distribution on legs reported by Yew et al. was not [18]. We confirmed these results presented by Yew et al. by analyzing samples of dissected legs. The sample preparation protocol had to be modified to obtain sufficient signal intensity. Due to the hairy surface of the fly leg and its very small outer diameter (in a range of $25 \mu \mathrm{m}$ to $60 \mu \mathrm{m}$ in the case of the tarsi and tibia), epoxy glue could not be applied to the whole length. Therefore, only the femur end of the leg was fixed to the MALDI plate. Allowing leg flexibility could have resulted - in combination with an ablation plume - in slight movement during measurement. Nevertheless, realistic MSI data were obtained (Fig. 2) after mass recalibration (Fig. 2c,f), and 7,11-HD and 7,11-ND were shown to exhibit a similar lateral distribution on both tarsi and tibia, with the highest intensity for 7,11-ND.

Legs and wings were also prepared on the normal MALDI target (Fig. S6). Reconstructed images for the lateral distribution of both 7,11-HD and 7,11-ND on wings show similar signal intensities to those on legs, with the highest signals measured on the tarsi. 




MALDI spectra show a pronounced mass shift in both the blue and the red regions (see Fig. 2e). Without mass spectra recalibration [24], the signals are shifted even up to $1 \mathrm{Da}$, and signal annotation is complicated. On the other hand, laser irradiation of an individual spot results in a correct mass readout and no mass shift is observable (Fig. S7). We assume that the hairy structures (Fig. S2f) on legs accumulate an electric charge after prolonged UV-laser irradiation during MSI, and even when legs are mounted on a metallic target or on carbon tape, the charge imposed in a high vacuum barely dissipates (data not shown).

\section{LDI-TOF MSI of male pheromones on virgin $D$. melanogaster males}

Another set of MSI experiments focused on the malespecific sex pheromones cVA [8] and CH503 [18], and their distribution on the cuticles of virgin male flies. Measurements were carried out in LDI-TOF positive mode (without the LiDHB matrix) because the oxygenated nature of these compounds resulted in efficient ionization, unlike the unsaturated hydrocarbons represented by female pheromones. In virgin flies, both pheromones were found only in males and both were similarly distributed exclusively on the lower part of the male abdomen (Fig. 3).

Identical LDI experiments in which we fixed virgin males and females on the MALDI target on their dorsal side to visualize $\mathrm{CH} 503$ and cVA at the ventral anogenital region showed the presence of both pheromones only in virgin males (Fig. 4).

Similar results confirming the presence of cVA solely in male flies were obtained by GC-MS analysis of paper material used for rubbing the anogenital region of virgin males (Fig. S8). Surprisingly, we did not identify any $\mathrm{CH} 503$ in our GC-MS spectra. Its relatively high molecular weight and its low thermal stability under GC conditions may have caused compound degradation.

\section{Transfer of male-specific sex pheromones to females during copulation}

We next tested whether our analysis could also be used to document the described transfer of pheromones from male to females during mating. In order to perform imaging experiments with mated males and females, one sixdays-old virgin male and female fly (both reared individually) were placed into a plastic tube and kept together till the end of their copulation. The act of copulation and also 


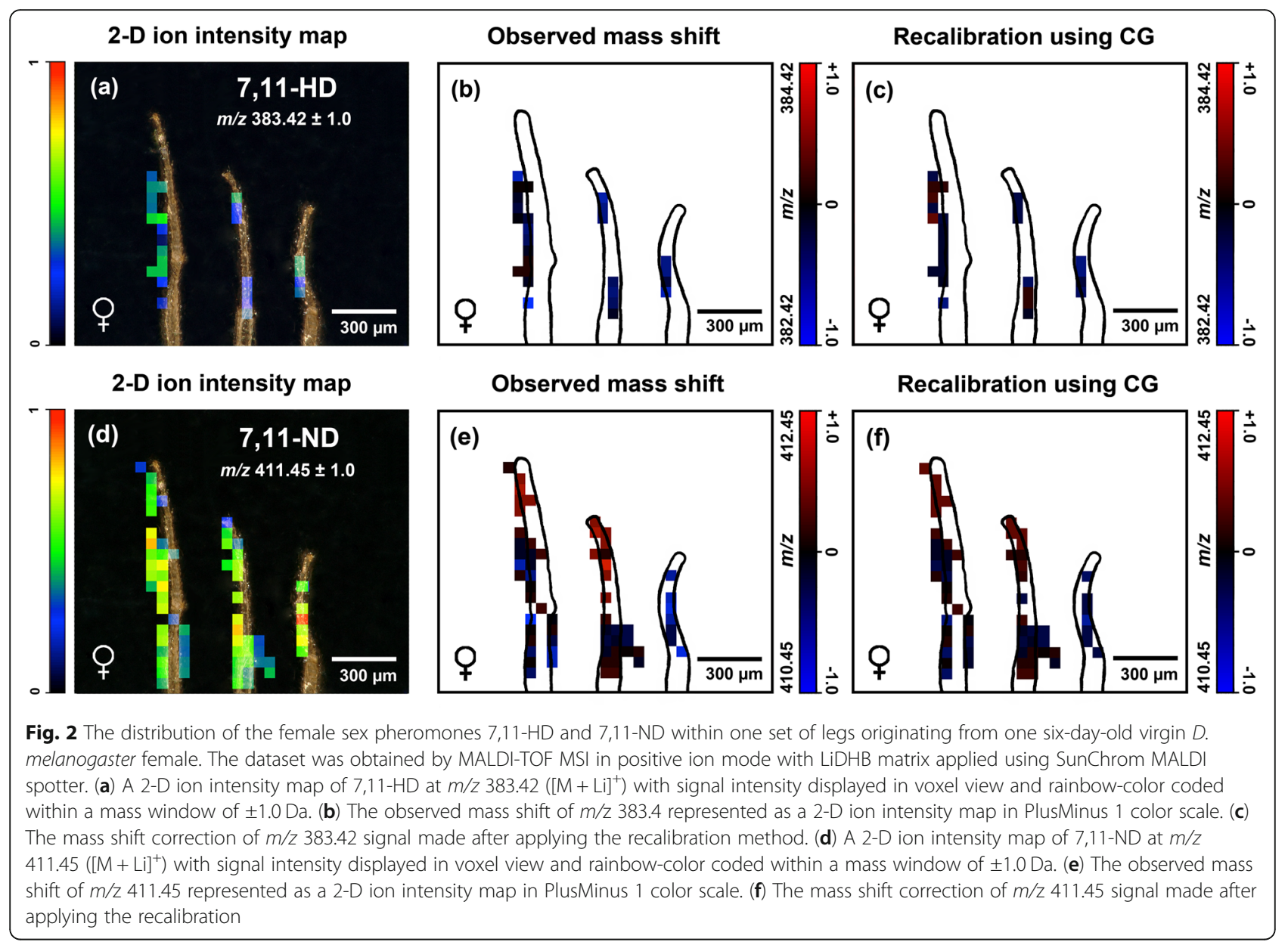

the courtship were recorded on camera, and the obtained footage was studied afterwards. MSI experiments with mated flies were carried out in LDI-TOF mode. Unlike virgin females (Fig. 3a), mated females had a strong signal of cVA and $\mathrm{CH} 503$ on both the dorsal (Fig. 5) and the ventral (Figure S9) sides of the wing. Although the ventral signal was expected, due to its close contact with the male anogenital region during mating, the presence of cVA on the female abdomen and the male thorax (Fig. 5a) was striking.

\section{GC-MS analyses of female and male hexane washes}

The fast hexane extraction of dissected wings, legs and the remaining body with an internal standard added to the mixture provided rich extracts from each sex; all were subsequently analyzed using GC-MS. The identity of described compounds is based on typical fragmentation patterns of EI-MS spectra, and 31 compounds were quantified in female samples (Table S3, Fig. S10). Pheromone dienes were found to occur in similar quantities in all dissected tissues. Wings and especially the body contain a level of saturated compounds that is likely related to the protection they provide against water evaporation (Fig. 6a). However, if a surface area of individual samples of wings, legs and rest of the body (Table S2) is assigned with the quantitative GC-MS data it revealed a disparity between samples. The legs with smallest area shown highest concentration of dienes per squere millimeter of sample surface (Fig. 6b) and the wings and body exhibit approximately 3 times and 2.5 times lower concentration, respectively. No trace of cVA was detected in any tissues.

To deduce the distribution of cVA from MSI data, we analyzed male extracts. As expected, cVA was present on male body parts only (Fig. S11). Other components identified included a series of saturated and 2-methyl branched saturated hydrocarbons from C21 to C31, and monoenic hydrocarbons from C21 to C29. No dienes or CH503 were detected.

\section{Discussion}

Our MALDI MSI experiments revealed a distribution of female pheromones 7,11-HD and 7,11-ND on female wings, with the strongest signals at the wing base. We also observed signals from 7,11-HD and 7,11-ND in the wing blade area (Fig. 1a). This symmetrical pattern, which was found on both the dorsal and the ventral sides of the wing, might be explained by the presence of 


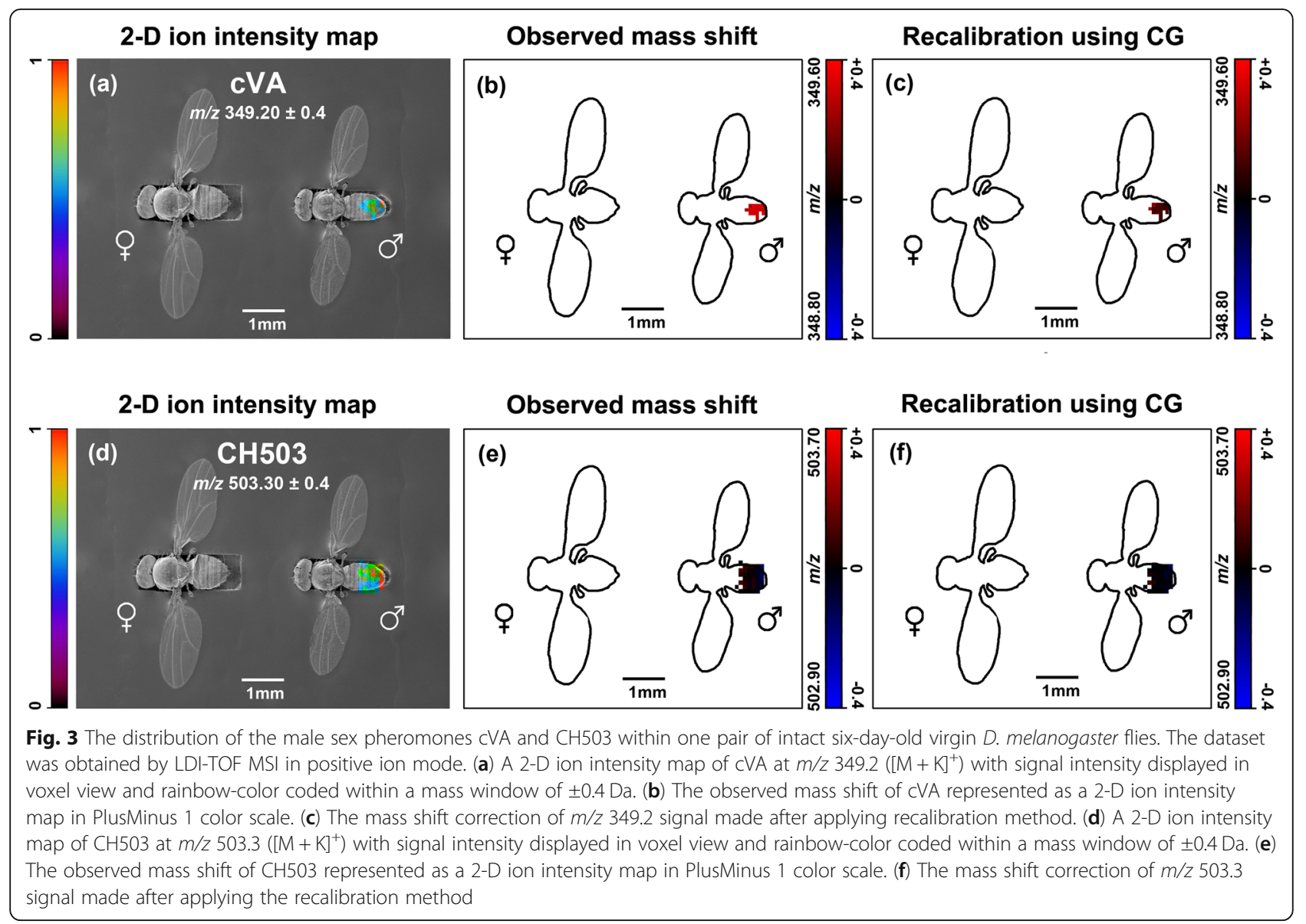

capillaries connecting pheromone gland outlets localized in the wing base region with the rest of the wing. Deployment of pheromones onto the wing blade area could lead to increases in spreading of the pheromones. In contrast to the previous explanation, which is the spread of female pheromones to the wing blade via the legs, is reflected in the symmetry of the pheromone distribution, that is, the presence of pheromones on both the dorsal and the ventral wing sides. As a similar distribution of corresponding dienes at the wing base was observed on the wings of the grayflesh fly (Neobellieria bullata) [21], we believe this distribution may be common in other Hymenoptera.

On the other hand, imaging experiments using whole Drosophila flies showed weaker signals compared to experiments using dissected wings. Because the base of the dissected wings is never directly connected to the metal MALDI target, poor contact may explain the weak signals in female HCs; electric charging, poor ion extraction and trapped ions in this region, all of which result from poor contact, are known to lower signal intensities in MS spectra [22]. In addition, the oxygenated compounds in the wing base region most likely increased after the LiDHB matrix was sprayed on the fly. (When matrix is sprayed on a fly prior to drying, the extraction of lipids -- especially from deeper cuticle layers -- is facilitated.) [24]. Therefore, the poor ion extraction in combination with preferential ionization of oxygenated compounds (presented on the surface) against hydrocarbons emerged in very low intensity of 7,11-HD and 7,11ND ions in MS spectra.

Legs have been reported to be bearing the highest amount of dienes in Drosophila [18]. Our MSI experiments confirmed this finding: both tarsi and tibia showed high amounts of dienes. The MSI data were supported by GC-MS data from hexane extracts of dissected fly parts.

Monoenes and dienes are biosynthesized from fatty acids of different chain length by the enzyme delta- 9 fatty acid desaturase [26, 27]. Formed monoenic acids are elongated/decarboxylated to odd-carbon-number monoenes in both males and females. Female flies possess the desatF desaturase working on monoenic acid providing dienic acids on carbon, which are elongated/ decarboxylated to odd-carbon-number dienes [28]. The second desaturation step is under control of Antennapedia and Bithorax gene complexes, and a factor originating from prothorax has a great influence on the production of dienes in abdominal segments 4 to 5 . The 


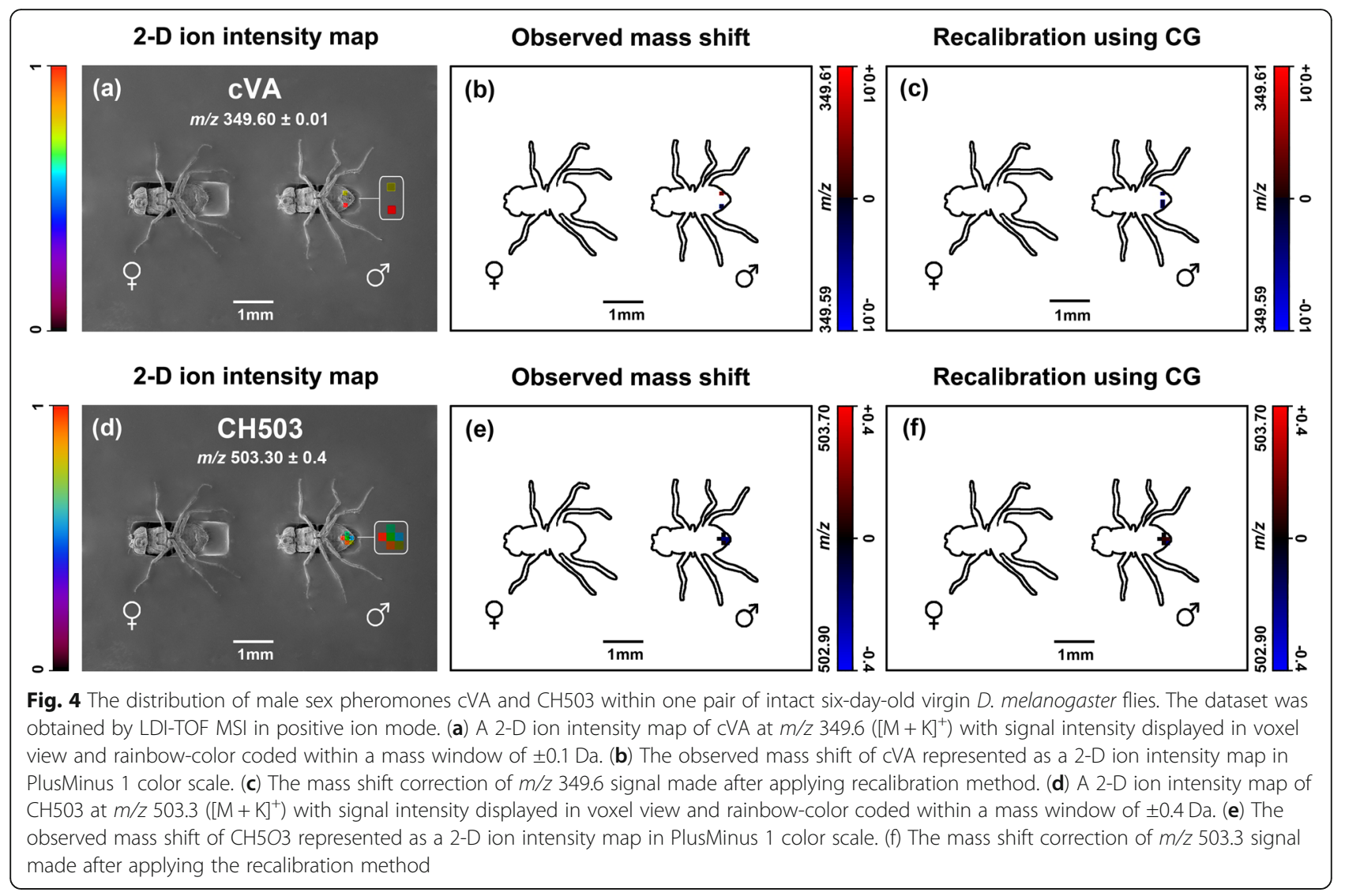

dienes are most likely produced in oenocytes [29]. Synthetized hydrocarbons are afterwards transported by lipophorines circulating in hemolymph. This can explain the ubiquitous occurrence of dienes in all parts of the female body. On the other hand, that male sex pheromones are mostly localized in specialized parts of the body, such as pheromonal glands, is well known from Lepidoptera. In Drosophila, female sex pheromones are recognized by specific receptors localized on male legs [30]. Dienes represent overall signal of femininity to males.

Our MSI experiments focused on male sexual pheromones showed a similar distribution of cVA and $\mathrm{CH} 503$ (Figs. 3 and 4). As cVA and CH503 are co-localized, they most probably occurred in a mixture. Our conclusions are supported with findings published by Yew et al. [18] showing by in situ MS profiling that cVA and CH503 are co-localized at the tip of the male fly ejaculatory bulb, in which cVA is stored [25] and in which also the last biosynthetic step of cVA takes place [31]. Accordingly, we suggest that $\mathrm{CH} 503$ might be also stored in the ejaculatory bulb. $\mathrm{CH} 503$ biosynthesis is under control of the Bond gene, which is expressed on the reproductive organ epithelium [32], and $\mathrm{CH} 503$ can be profiled from this area. However, based on recently published work by Niehoff et al. [33] and Khalil et al. [34], CH503 seems to be more widespread than $\mathrm{cVA}$ and can be detected in the entire posterior area of the male flies.

The courtship videos (Fig. 7) revealed that a male not only comes into contact with the female abdominal tip during copulation, but also probes the female with its legs (Fig. 7a) and proboscis (Fig. 7c) as described by Guiraudie-Capraz [31]. Those are the parts of the female - wings and abdomen - that according to our analysis, after courtship carries the male-specific pheromones. Additional evidence that the male could use its legs to transfer specific biologically active compounds comes from Yew et al., who documented the transfer of female pheromones to male legs after copulation [18].

In addition, our MSI results of whole Drosophila flies also demonstrated that both male pheromones are spread over the entire male abdomen (especially on the dorsal side). Such coverage could be achieved partly by the passive diffusion of pheromones in a layer of cuticular lipids but more likely by using the hindmost pair of legs.

MALDI MSI using the LiDHB matrix exhibits a dramatic mass shift that originates from laser-induced electric charging/discharging on the surface of the flies. Using the computational recalibration approach, mass shift can be considerably reduced. Nevertheless, ionization is sometimes suppressed (as we observed on the female fly body), possibly by the presence of oxygenated compounds on the 


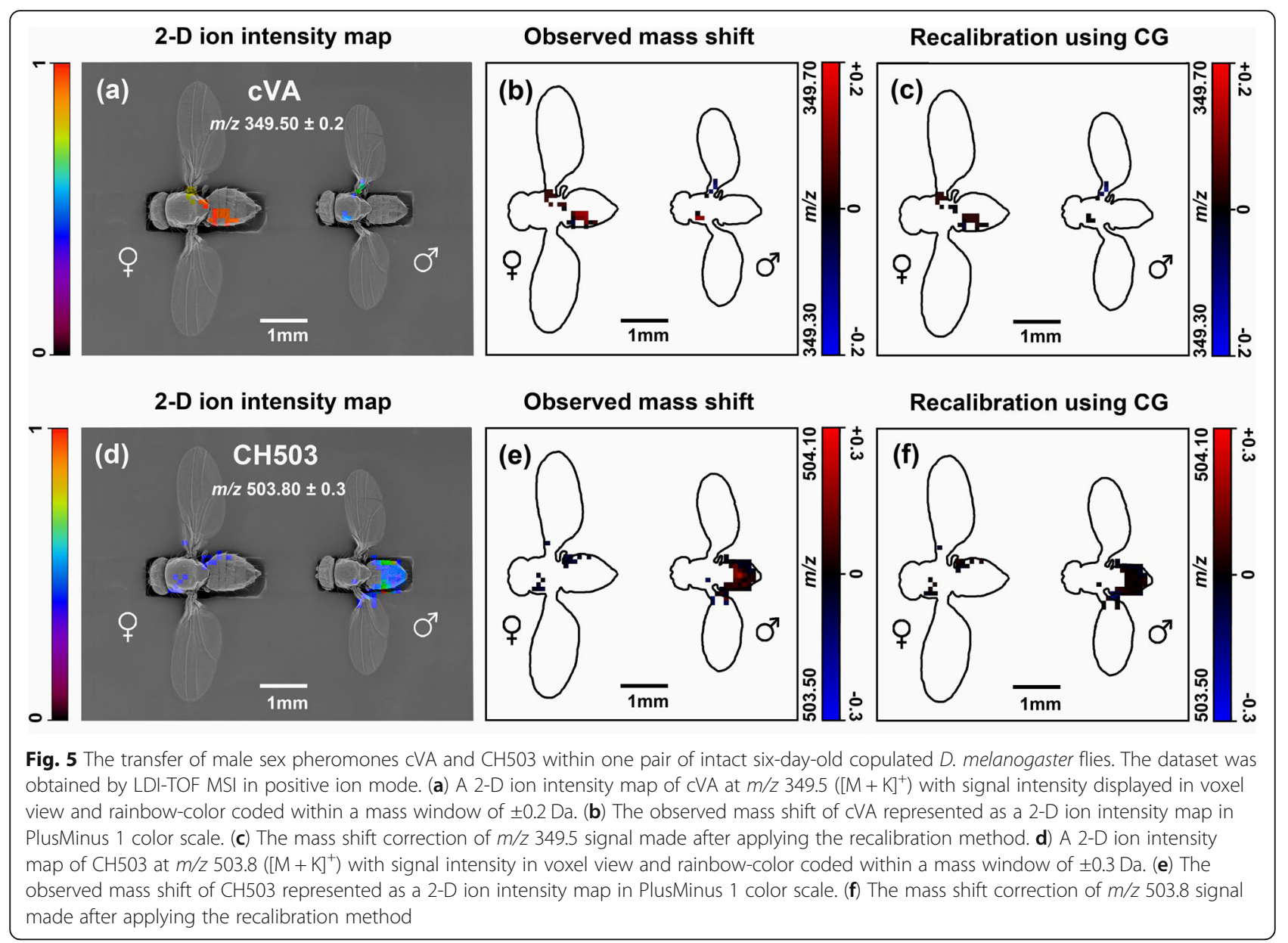

fly cuticle, likely triacylglycerols. Using LiDHB under high vacuum is the only known way to image saturated hydrocarbons, as has been shown in bees [35]. Another method for cuticle profiling, developed in Dreisewerd's laboratory and named FALDI [36], uses UV-LDI mass spectrometry on orthogonal-flight instrument geometry; however, as yet this method is unable to ionize saturated hydrocarbons. The ion yield strongly depends on the pressure in the source, and in commercial instruments, such pressure is not easily controlled.

\section{Conclusion}

A dedicated aluminum MALDI target with imprinted shaped grooves for male and female flies allowed us to analyze in LDI or MALDI mode the distribution of pheromones on both the dorsal and the ventral sides of Drosophila flies. The distribution of hydrocarbons represented by female pheromones 7,11-HD and 7,11-ND was studied on virgin and mated female flies via MALDI-TOF MSI with a LiDHB matrix. Signals from the pheromones revealed a symmetrical pattern for the left and right female wings, with the strongest signals located at the base of the wings. No difference was observed between virgin and mated female flies, and no signals of 7,11-HD or 7,11-ND were observed on virgin or mated male flies. The distribution of male cVA and $\mathrm{CH} 503$ pheromones, also visualized in LDI-TOF MSI, on virgin males was identical. These pheromones are exclusively present on the abdominal tip and the anogenital region. The situation was slightly different in the MSI of mated females. MSI clearly indicates that both compounds are transferred simultaneously during copulation onto the female. Moreover, our experiments suggest which part of the female body males transfer their pheromones to during mating.

As the transfer of male pheromones significantly reduces female sexual attractiveness to other males [19], these imaging experiments based on 2-D visualization confirm the role of cVA and $\mathrm{CH} 503$ as male-transferred anti-attractants. Results from all MSI experiments were supported by GC-MS analyses of hexane extracts of specific parts of both male and female flies. GC-MS results confirmed the presence of 7,11-HD or 7,11-ND in the extracts of female wings and also that of cVA in samples prepared by rubbing the anogenital region of males and the abdominal tips of mated females. The presence of both female pheromones and cVA in the MS spectra 

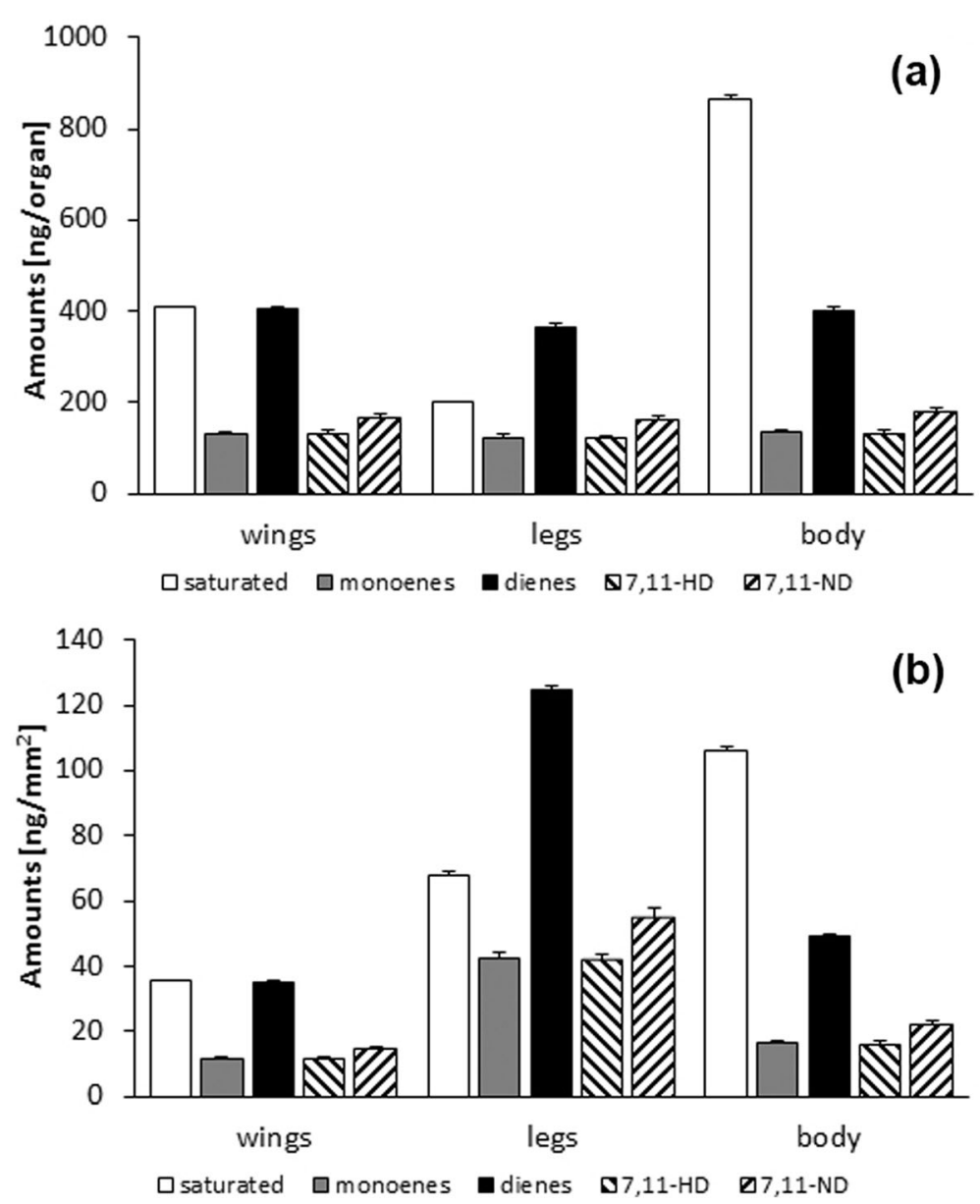

Fig. 6 An abundance of compounds is detected in hexane washes of dissected wings, legs and the remaining body parts. (a) Amounts are calculated using internal standard (b) The amounts divided by surface areas are reported in Table $S 2$. $N=5$. For compound identification, see Table S3
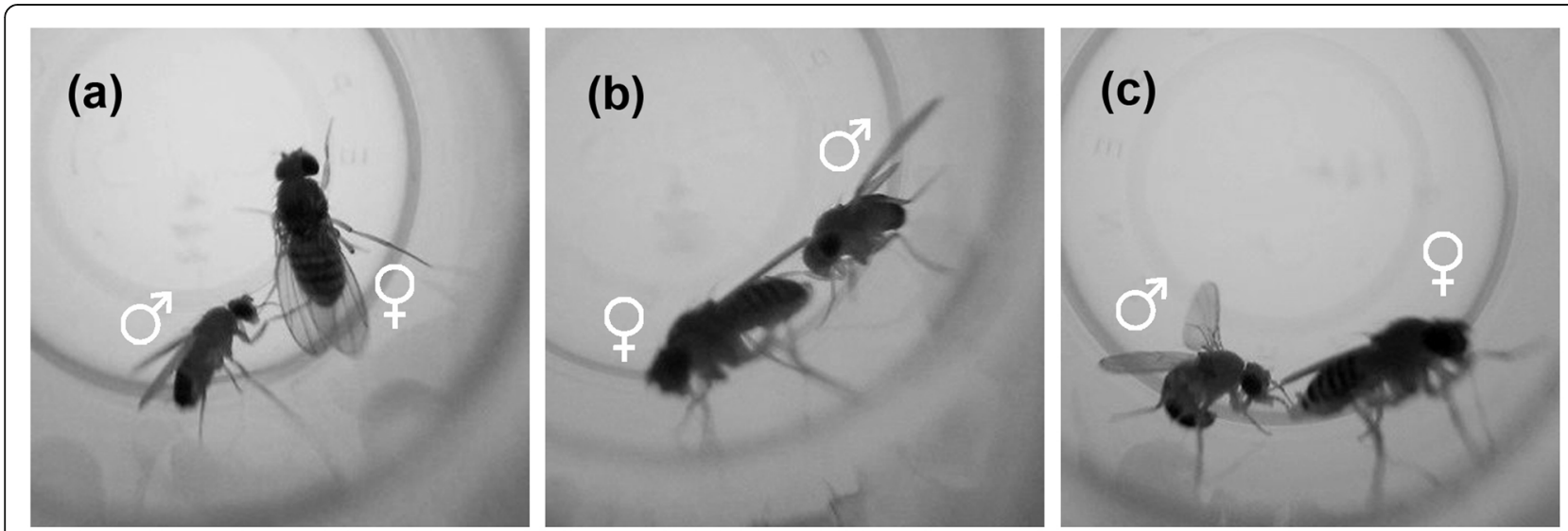

Fig. 7 Male courtship is a potential means by which pheromones are transferred. (a) The male fly taps with legs on the side of the female abdomen. (b) The male fly approaches the female from behind and taps on the upper part of the female abdomen, which is located under the wings. (c) The copulation attempt is preceded by the male tapping on the female anogenital region 
was confirmed by analyzing standards under the same experimental conditions (Figs. S8 and S12). That $\mathrm{CH} 503$ was not, however, found in the GC-MS spectra - most likely it was degraded under the GC conditions - highlights the sensitivity and usefulness of MALDI-TOF.

\section{Supplementary information}

Supplementary information accompanies this paper at https://doi.org/10. 1186/s40850-020-00053-7.

Additional file 1: Table S1. D. melanogaster (Canton-S) recipe of $1000 \mathrm{~mL}$ fly food Table S2. Surface area of different body parts of six-day-old virgin female D. melanogaster (Canton-S) fly Table S3. List of compounds identified from GC-MS analysis of wings, legs and rest of the body of sixday-old virgin female $D$. melanogaster fly hexane extracts. (See file "Supplementary data D. melanogaster female body parts GCMS analysis.Xls $x^{\prime \prime}$ ). Figure S1. Optical and scanning electron microscopy images of six-day-old virgin female and male D. melanogaster flies. (a) Female fly freshly fixed onto a dedicated MALDI target (b-d) Female fly after 6 hours of drying in desiccator (e) Female fly after 2 days of drying (f) Male fly after 2 days of drying ( $\mathrm{g}-\mathrm{h}$ ) Detail on male abdominal tergite deformed by drying Figure S2. Scanning electron microscopy images of six-day-old virgin D. melanogaster flies covered with LiDHB matrix using airbrush $(a, b, c)$ and SunChrom MALDI spotter (d). (a) Female thorax (b) Female abdomen (c,d) Female wing. Figure S3. Distribution of the female sex pheromone 7,11ND on one pair of six-day-old copulated D. melanogaster flies. Dataset was obtained by MALDI-TOF MSI in positive ion mode with the LiDHB matrix. (a) 2-D ion intensity map of 7,11-ND at $\mathrm{m} / \mathrm{z} 411.6([\mathrm{M}+\mathrm{Li}]+)$ ) with signal intensity displayed in voxel view and rainbow-color coded within a mass window of $\pm 0.1 \mathrm{Da}$. (b) Observed mass shift of 7,11- ND represented as a 2$\mathrm{D}$ ion intensity map in PlusMinus 1 color scale. (c) The mass shift correction of $\mathrm{m} / \mathrm{z} 411.6$ after applying the recalibration method. Figure S4. El-MS chromatogram and spectra of hexane extract from 10 pairs of wings from virgin D. melanogaster females. (a) El/MS chromatogram with focus on the retention time of female pheromones 7,11-HD and 7,11-ND. (b) Female sex pheromone 7,11-HD at $\mathrm{m} / \mathrm{z}$ 376.4. (c) Female sex pheromone 7,11-ND at $\mathrm{m} /$ z 404.4. Figure S5. Visualization of unknown masses evincing symmetrical distribution within the region of the wing base on one six-day-old copulated female D. melanogaster fly. Dataset was obtained by MALDI-TOF MSI in positive ion mode with the LiDHB matrix. (a) 2- D ion intensity map of $m / z 379.6$ with signal intensity displayed in voxel view and rainbow-color coded within a mass window of $\pm 0.2 \mathrm{Da}$. (b) 2-D ion intensity map of $\mathrm{m} / \mathrm{z}$ 419.6 with signal intensity displayed in voxel view and rainbow-color coded within a mass window of \pm 0.2 Da. (c) 2-D ion intensity map of $m / z 511.7$ with signal intensity displayed in voxel view and rainbow-color coded within a mass window of $\pm 0.2 \mathrm{Da}$. Figure S6. Visualization of the female pheromones 7,11-HD and 7,11-ND within a six-day-old virgin D. melanogaster female fly wings and one set of legs using MALDI-TOF. Figure S7. MALDI-TOF MS spectrum of female pheromones 7,11-HD and 7,11-ND acquired during analysis of a last tarsal segment (with claws) of D. melanogaster female third pair of legs. Analysis was performed in profiling mode. Figure S8. El/MS chromatogram and spectrum of hexane extract prepared by rubbing the abdomens and anogenital regions of 14 virgin D. melanogaster males using lipid-free paper. (a) El/MS chromatogram with a focus on the elution time of CVA. (b) MS signal of male sex pheromone CVA at $\mathrm{m} / \mathrm{z} 250.1[\mathrm{M}+\cdot \boldsymbol{-} \mathrm{CH} 3 \mathrm{COOH}]+\cdot$. Figure S9. Transfer of sexual pheromones CVA and $\mathrm{CH} 503$ from male to female during copulation was investigated via MALDI-MSI experiments with LiDHB matrix. Copulated pair of flies was analyzed on its ventral side with focus on anogenital area. Figure S10. Typical chromatogram obtained via GC-MS analysis of virgin D. melanogaster female hexane extracts.

Additional file 2. Supplementary data D. melanogaster female body parts GC-MS analysis.

\section{Abbreviations}

7,11-HD: (7Z,11Z)-Heptacosa-7,11-diene; 7,11-ND: (7Z,11Z)-Nonacosa-7,11diene; CG: Crystal growth ordering; CH503: 3-O-Acetyl-1,3-dihydroxyoctacosa-11,19-diene; cVA: 11-cis-accenyl acetate; Da: Dalton DHB: 2,5-
Dihydroxybenzoic acid; GC-MS: Gas chromatography - mass spectrometry; LDI: Laser desorption ionization; LiDHB: Lithium 2,5-dihydroxybenzoate; MALDI-TOF: Matrix-assisted laser desorption/ionization - time of flight MS: Mass spectrometry; MSI: Mass spectrometry imaging; SEM: Scanning electron microscope

\section{Acknowledgements}

Financial support from the Max Planck Society for instruments is gratefully acknowledged. We would like to thank Frank Steiniger from

Elektronenmikroskopisches Zentrum in Jena, Germany for his help with SEM images and also Sybille Lorenz and Kerstin Weniger, Anne Späthe, all from MPI-CE, for measuring samples on GC-MS. We would like to thank Emily Wheeler, Boston, for editorial assistance.

\section{Heading}

2-D surface distribution of sex pheromones within the whole Drosophila fly by mass spectrometry imaging.

\section{Authors' contributions}

FK designed and performed (MA) LDI experiments. FK and AS wrote the manuscript. PK recalibrated MSI datasets and generated 2-D ion intensity maps. AS, MK, BSH and SB revised the manuscript. All authors read and approved the final manuscript.

\section{Funding}

Financial support instrumentation, supply, and salary was provided by the Max Planck Society.

\section{Availability of data and materials}

The datasets used and/or analyzed during the current study are stored at Mass spectrometry/Proteomics Research Group at Max Planck Institute for Chemical Ecology, Jena, Germany and are available from the corresponding author on reasonable request.

\section{Ethics approval and consent to participate}

Not applicable.

\section{Consent for publication}

Not applicable.

\section{Competing interests}

The authors declare that they have no competing interests.

\section{Author details}

${ }^{1}$ Max Planck Institute for Chemical Ecology, Hans-Knöll-Strasse 8, D-07745 Jena, Germany. ${ }^{2}$ Chair for Bioinformatics, Friedrich Schiller University Jena, Q2 Ernst-Abbe-Platz 2, D-07743 Jena, Germany.

Received: 13 December 2017 Accepted: 12 April 2020

Published online: 07 May 2020

\section{References}

1. Cook RM. The courtship tracking of Drosophila melanogaster. Biol Cybern. 1979;34:91-106.

2. Schäffel F, Willmund R. Visual signals in the courtship of Drosophila melanogaster: mutant analysis. J Insect Physiol. 1985;31:899-907.

3. Ewing AW, Bennet-Clark HC. The courtship songs of Drosophila. Behaviour. 1968;31:288-301.

4. Kyriacou CP, Hall JC. Circadian rhythm mutations in Drosophila melanogaster affect short-term fluctuations in the male's courtship song. Proc Natl Acad Sci U S A. 1980;77:6729-33.

5. Kyriacou CP, Hall JC. Interspecific genetic control of courtship song production and reception in Drosophila. Science. 1986;232:494-7.

6. Ejima A, Griffith LC. Courtship initiation is stimulated by acoustic signals in Drosophila melanogaster. PLoS One. 2008;3(9):E3246-55.

7. Tootoonian S, Coen P, Kawai R, Murthy M. Neural representations of courtship song in the Drosophila brain. J Neurosci. 2012;32:787-98.

8. Jallon JM. A few chemical words exchanged by Drosophila during courtship and mating. Behav Genet. 1984;14:441-78.

9. Dweck HKM, Ebrahim SAM, Thoma M, Mohamed AAM, Keesey IW, Trona F, Lavista-Llanos S, Svatoš A, Sachse S, Knaden M, Hansson BS. Pheromones 
mediating copulation and attraction in Drosophila. PNAS. 2015;112(21): E2829-35.

10. Lin HH, Cao DS, Sethi S, Zeng Z, Chin JS, Chakraborty TS, Shepherd AK, Nguyen CA, Yew JY, Su CY, Wang JW. Hormonal modulation of pheromone detection enhances male courtship success. Neuron. 2016;90(6):1272-85.

11. Stocker RF. The organization of the chemosensory system in Drosophila melanogaster: a review. Cell Tissue Res. 1994;275:3-26.

12. Bray S, Amrein H. A putative Drosophila pheromone receptor expressed in male-specific taste neurons is required for efficient courtship. Neuron. 2003; 39:1019-29.

13. Butterworth FM. Lipids of Drosophila: a newly detected lipid in the male. Science. 1969;163(3873):1356-7.

14. Bartelt RJ, Schaner AM, Jackson LL. Cis-Vaccenyl acetate as an aggregation pheromone in Drosophila melanogaster. J Chem Ecol. 1985;11(12):1747-56.

15. Brieger G, Butterworth FM. Drosophila melanogaster: identity of male lipid in reproductive system. Science. 1970;167(3922):1262.

16. Ejime A, Smith BP, Lucas C, van der Goes van Naters W, Miller CJ, Carlson JR, Levine JD, Griffith LC. Generalization of courtship learning in Drosophila is mediated by cis-vaccenyl acetate. Curr Biol. 2007:17(7):599-605.

17. Laturney M, Billeter JC. Drosophila melanogaster females restore their attractiveness after mating by removing male anti-aphrodisiac pheromones. Nat Commun. 2016;7:1-11.

18. Yew JY, Dreisewerd K, Luftmann H, Müthing J, Pohlentz G, Kravitz EA. A new male sex pheromone and novel cuticular cues for chemical communication in Drosophila. Curr Biol. 2009;19:1245-54.

19. Antony C, Davis TL, Carlson DA, Pechine JM, Jallon JM. Compared behavioral responses of male Drosophila melanogaster (Canton-S) to natural and synthetic aphrodisiacs. J Chem Ecol. 1985;11:1617-29.

20. Svatoš A. Mass spectrometric imaging of small molecules. Trends Biotechnol. 2010;28(8):425-34.

21. Vrkoslav V, Muck A, Cvačka J, Svatoš A. MALDI imaging of neutral cuticular lipids in insects and plants. J Am Soc Mass Spectrom. 2010;21:220-31.

22. Kaftan F, Vrkoslav V, Kynast P, Kulkarni P, Böcker S, Cvačka J, Knaden M, Svatoš A. Mass spectrometry imaging of surface lipids on intact Drosophila melanogaster flies. J Mass Spectrom. 2014;49:223-32.

23. Cvačka J, Svatoš A. Matrix-assisted laser desorption/ionization analysis of lipids and high molecular weight hydrocarbons with lithium 2,5dihydroxybenzoate matrix. Rapid Commun Mass Spectrom. 2003;17:2203-7.

24. Kulkarni P, Kaftan F, Kynast P, Svatoš A, Böcker S. Correcting mass shifts: a lock mass-free recalibration procedure for mass spectrometry imaging data. Anal Bioanal Chem. 2015;407:7603-13.

25. Zawistowski S, Richmond RC. Inhibition of courtship and mating of Drosophila melanogaster by the male-produced lipid, cis-vaccenyl acetate. J Insect Physiol. 1986;32:189-92.

26. Dallerac R, Labeur C, Jallon JM, Knipple DC, Roelofs WL, Wicker-Thomas C. A $\Delta 9$ desaturase gene with a different substrate specificity is responsible for the cuticular diene hydrocarbon polymorphism in Drosophila melanogaster. PNAS. 2000;97:9449-54.

27. Labeur C, Dallerac R, Wicker-Thomas C. Involvement of desat1 gene in the control of Drosophila melanogaster pheromone biosynthesis. Genetica. 2002; 114(3):269-74

28. Chertemps T, Duportets L, Labeur C, Ueyama M, Wicker-Thomas C. A female-specific desaturase gene responsible for diene hydrocarbon biosynthesis and courtship behaviour in Drosophila melanogaster. Insect Mol Biol. 2006:15(4):465-73.

29. Wicker-Thomas C, Jallon JM. Control of female pheromones in Drosophila melanogaster by homeotic genes. Genet Res Camb. 2001;78:235-42.

30. Thistle R, Cameron P, Ghorayshi A, Dennison L, Scott K. Contact Chemoreceptors Mediate Male-Male Repulsion and Male-Female Attraction during Drosophila Courtship. Cell. 2012;149:1140-51.

31. Guiraudie-Capraz G, Pho DB, Jallon JM. Role of the ejaculatory bulb in biosynthesis of the male pheromone cis-vaccenyl acetate in Drosophila melanogaster. J Integr Zool. 2007;2:89-99.

32. Ng WC, Chin JS, Tan KJ, Yew JY. The fatty acid elongase bond is essential for Drosophila sex pheromone synthesis and male fertility. Nat Commun. 2015;6:8263

33. Niehoff AC, Kettling H, Pirkl A, Chiang YN, Dreisewerd K. Analysis of Drosophila lipids by matrix-assisted laser desorption/ionization mass spectrometric imaging. Anal Chem. 2014;86:11086-92.

34. Khalil SM, Pretzel J, Becker K, Spengler B. High-resolution AP-SMALDI mass spectrometry imaging of Drosophila melanogaster. IJMS. 2017:416:1-19.
35. Nunes TM, Mateus S, Favaris AP, Amaral MFZJ, von Zuben LG, Clososki GC Bento JMS, Oldroyd BP, Silva R, Zucchi R, Silva NB, Lopes NP. Queen signals in a stingless bee: suppression of worker ovary activation and spatial distribution of active compounds. Sci Rep. 2014;4:7449.

36. Yew JY, Soltwisch J, Pirkl A, Dreisewerd K. Direct laser desorption ionization of endogenous and exogenous compounds from insect cuticles: practical and Methodologic aspects J. Am Soc Mass Spectrom. 2011;22:1273-84.

\section{Publisher's Note}

Springer Nature remains neutral with regard to jurisdictional claims in published maps and institutional affiliations.
Ready to submit your research? Choose BMC and benefit from:

- fast, convenient online submission

- thorough peer review by experienced researchers in your field

- rapid publication on acceptance

- support for research data, including large and complex data types

- gold Open Access which fosters wider collaboration and increased citations

- maximum visibility for your research: over $100 \mathrm{M}$ website views per year

At $\mathrm{BMC}$, research is always in progress.

Learn more biomedcentral.com/submissions 